\title{
Nutrient Requirements of Nonhuman Primates: Second Revised Edition
}

Reviewed by Joseph Knapka, PhD

\begin{abstract}
The National Research Council's (NRC's) report, Nutritional Requirements of Nonhuman Primates, is an outstanding reference not only for the nutritional requirements of nonhuman primates but also for the nutritional management of various captive nonhuman primate species. A committee of 11 individuals who are considered to be experts in nonhuman primate nutrition and biology prepared this second edition of the report. The result is a publication that is the most extensive of the NRC series of reports on
\end{abstract} domestic animal nutrition, in that it is essentially a textbook on nonhuman primate nutrition rather than just a source of information on the estimated nutrient requirements of a variety of species.

This report contains a comprehensive review of the current literature related to nonhuman primate nutrition. It is very well written and illustrated with numerous tables and figures that explain the most complex nutritional principles in a manner that is relatively easy to read and understand. It is therefore the most important contribution ever made to nonhuman primate nutrition, and it should be a welcome addition to the personal libraries of nonhuman primate colony managers involved with biomedical research and zoos, veterinarians with nonhuman primate responsibilities, and students of nonhuman primate nutrition everywhere.

The "Energy", "Carbohydrates and Fiber", "Protein", "Fats and Fatty Acids",
"Minerals", "Vitamins", and "Water" chapters are similar to those that are traditionally included in similar NRC reports. The metabolic role of each nutrient is discussed, nutrient deficiency and toxicity signs are described, and the documentation supporting the estimated nutrient requirements is presented. The estimated nutrient requirements are presented in two easy-to-read tables. In one table, estimated requirements are presented for nine nonhuman primate species consuming purified and Price: $\$ 47.96$ ISBN: 0309069890 and the differences in anatomy and function of the digestive tracts of the various species of nonhuman primates.

A wide range of topics that must be considered in the nutritional management of nonhuman primate colonies is presented in a chapter entitled "Pathophysiologic and LifeStage Considerations”. Examples of topics discussed in this chapter include rearing of juvenile animals, nutrition and aging, obesity, and the nutritional management of animals with pathologies, such as diabetes. A chapter entitled "Food as a Component of Environmental Enhancement" provides excellent information about strategies for the use of various foods as a means to enrich the captive nonhuman primate's psychological well-being to comply with federal regulations.

As in most NRC reports of this nature, a table is included listing the nutrient composition of feed ingredients used to manufacture feeds for the species of interest. This table has been expanded in this report to include most of the succulent and live feedstuffs that are used as supplements in numerous captive nonhuman primate colonies. This list is very extensive, and it should be considered a primary source of information about the nutrient composition of these various foodstuffs. Anyone with an interest in the amount of nutrients consumed by nonhuman primates being fed various supplemental foodstuffs should obtain a copy of this report.

Softcover copies of this report are available from The National Academies Press (NAP), Washington, DC, for $\$ 47.96$. The report can be read online at http:// www.nap.edu./catalog/9826.html and is available in PDF format for $\$ 36.00$.

Knapka is a consultant in laboratory animal nutrition in Brookeville, MD. 